\title{
A criterion for extending morphisms from open subsets of smooth fibrations of algebraic varieties ${ }^{\star}$
}

\author{
Vassil Kanev \\ Dipartimento di Matematica e Informatica, Università di Palermo, Via Archirafi 34, 90123 \\ Palermo, Italy
}

\begin{abstract}
Given a smooth morphism $Y \rightarrow S$ and a proper morphism $P \rightarrow S$ of algebraic varieties we give a sufficient condition for extending an $S$-morphism $U \rightarrow P$, where $U$ is an open subset of $Y$, to an $S$-morphism $Y \rightarrow P$, analogous to Zariski's main theorem.

Keywords: extending a morphism, Zariski's main theorem, smooth fibration, fiberwise birational morphism

2010 MSC: 14A10, 14D06
\end{abstract}

\section{Introduction}

A well-known fact is that every morphism from an open subset of a nonsingular algebraic curve to a complete algebraic variety may be extended to a morphism of the curve. This is proved by considering the closure of the graph of the map in the product of the curve and the variety and proving that the projection of the closure to the curve is an isomorphism by means of Zariski's main theorem. It is desirable to generalize this extension property to families of curves, with a view toward applications to moduli problems. Trying to apply the same argument to families of curves one encounters the following problem. Given two families of curves $g: X \rightarrow S, h: Y \rightarrow S$, where $h$ is smooth, and a commutative diagram of morphisms as in (1) below, such that $f$ is finite and surjective, and $f_{s}: g^{-1}(s) \rightarrow h^{-1}(s)$ is birational for every $s \in S$, is it true that $f$ is an isomorphism? If $g$ and $h$ were proper and if the scheme-theoretic fibers of $g$ were reduced this would follow from Proposition 4.6 .7 (i) of [1]. The latter condition, however, might be difficult to verify. In fact a weaker condition on $g$ suffices to conclude that $f$ is an isomorphism. Before we formulate one

*This work was supported by Università di Palermo (research project 2012-ATE-0446). Manuscript accepted for publication in Journal of Pure and Applied Algebra, https://doi.org/10.1016/j.jpaa.2020.106553

(c) $\langle 2020\rangle$. This manuscript version is made available under the CC-BY-NC-ND 4.0

license http://creativecommons.org/licenses/by-nc-nd/4.0/

Email address: vassil.kanev@unipa.it (Vassil Kanev) 
of our results let us recall that a morphism of algebraic varieties $h: Y \rightarrow S$ over an algebraically closed field $k$ is smooth at $y \in Y$ if $h$ is flat at $y$ and $\operatorname{dim}_{k} \Omega_{Y / S}(y) \leq \operatorname{dim}_{y} Y_{h(y)}$. The following theorem holds.

Theorem 1. Let $X, Y$ and $S$ be algebraic varieties over an algebraically closed field $k$. Let $d$ be an integer $\geq 1$. Let

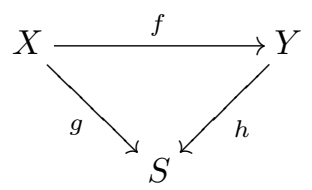

be a commutative diagram of morphisms such that:

a. $h: Y \rightarrow S$ is smooth of relative dimension $d$;

b. $f$ is finite and surjective;

c. for every $s \in h(Y)$ the fiber $g^{-1}(s)$ is irreducible and there is point $x \in$ $g^{-1}(s)$ such that $g$ is smooth at $x$;

d. for every $s \in g(X)=h(Y)$ the map $f_{s}: g^{-1}(s) \rightarrow h^{-1}(s)$ is birational.

Then $f: X \rightarrow Y$ is an isomorphism.

Theorem 1 is used in the proof of the following criterion for extension of morphisms.

Theorem 2. Let $Y, S$ and $P$ be algebraic varieties over an algebraically closed field $k$. Let $d$ be an integer $\geq 1$. Let $h: Y \rightarrow S$ be a smooth morphism whose nonempty fibers are irreducible of dimension d. Let $P \rightarrow S$ be a proper morphism. Let $U$ be an open subset of $Y$ such that $U \cap h^{-1}(s) \neq \emptyset$ for every $s \in h(Y)$. Let $\varphi: U \rightarrow P$ be an $S$-morphism. Let $\Gamma \subset U \times{ }_{S} P$ be its graph and let $X=\bar{\Gamma} \subset Y \times{ }_{S} P$ be its closure. Suppose that the projection $f: X \rightarrow Y$ has the property that $f^{-1}(y)$ is a finite set for every $y \in Y \backslash U$. Then there is a unique extension of $\varphi$ to $Y$ : an $S$-morphism $\tilde{\varphi}: Y \rightarrow P$ such that $\left.\tilde{\varphi}\right|_{U}=\varphi$.

We notice that if $S$ is irreducible and normal, then $Y$ is irreducible and normal as well, and the statement of Theorem 2 follows from Zariski's main theorem.

Under the assumptions of Theorem 1, when the relative dimension of $g$ : $X \rightarrow S$ is one, Theorem 9 of [2] applied to $\operatorname{Spec} \mathcal{O}_{X, x} \rightarrow \operatorname{Spec} \mathcal{O}_{S, s}$, where $x \in X$ is arbitrary, $s=g(x)$, yields that $g: X \rightarrow S$ is smooth, which implies that $f: X \rightarrow Y$ is an isomorphism. The proof of Theorem 9 of [2] needs however an additional assumption, as the author was kindly informed by Prof. J. Kollár (letter of January 21, 2019), namely Assumption 9.2 of Theorem 9 (ibid.) should hold for the completions $\left(\operatorname{Spec} \mathcal{O}_{X, x}\right)^{\wedge} \rightarrow\left(\operatorname{Spec} \mathcal{O}_{S, s}\right)^{\wedge}$. This condition might not hold under the assumptions of Theorem 1.

The paper is organized as follows. In Section 1 we prove Theorem 3, which is a result of commutative algebra of independent interest, and from which Theorem 1 and Theorem 2 are deduced. Given a local flat homomorphism of 
Noetherian local rings $(A, \mathfrak{m}) \rightarrow(R, \mathfrak{n})$ with regular fiber $R / \mathfrak{m} R$ and a finite homomorphism $R \rightarrow B$, Theorem 3 gives sufficient conditions which ensure that $R \rightarrow B$ is an isomorphism. The proof of this theorem is based on the key idea of J. Kollár by which Theorem 9 of [2] is proved, namely splitting the $R$ module $B$ into a direct sum of $R$ and another summand, then proving that this summand is zero. In Section 2 we give the proofs of Theorem 1 and Theorem 2.

Notation. If $\varphi: A \rightarrow B$ is a homomorphism of rings and $I \subset A, J \subset B$ are ideals we denote, following [3], $\varphi(I) B$ by $I B$ and $\varphi^{-1} J$ by $A \cap J$. If $\mathfrak{p} \subset A$ is a prime ideal, then $k(\mathfrak{p})$ is the quotient field of $A / \mathfrak{p}$. A variety over an algebraically closed field $k$ is a reduced separated, possibly reducible, scheme of finite type over $k$. A point of a variety is a closed point of the scheme. The maximal spectrum of a ring $A$ is denoted by $\operatorname{Spm} A$.

\section{The main theorem}

Theorem 3. Let $(A, \mathfrak{m})$ and $(R, \mathfrak{n})$ be Noetherian local rings, and let $A \rightarrow R$ be a local flat homomorphism. Suppose that $A$ is reduced. Suppose that:

a. $R \otimes_{A} k(\mathfrak{m})$ is a regular ring of dimension $n \geq 1$ and $k(\mathfrak{n})$ is separable over $k(\mathfrak{m})$;

b. $R \otimes_{A} k(\mathfrak{p})$ is a regular ring for every minimal prime ideal $\mathfrak{p}$ of $A$.

Set $S=\operatorname{Spec} A, s_{0}=\mathfrak{m}, Y=\operatorname{Spec} R, y_{0}=\mathfrak{n}$, and let $h: Y \rightarrow S$ be the associated morphism of affine schemes. Suppose there is a commutative diagram of morphisms of schemes

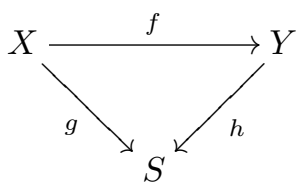

such that:

i. $f$ is finite and surjective;

ii. $X_{s_{0}}$ is irreducible and generically reduced;

iii. $g$ is flat at the generic point of $X_{s_{0}}$;

iv. if $\eta \in X_{s_{0}}$ and $\zeta=f(\eta) \in Y_{s_{0}}$ are the generic points of $X_{s_{0}}$ and $Y_{s_{0}}$ then $f^{\sharp}(\zeta): k(\zeta) \rightarrow k(\eta)$ is an isomorphism;

v. every irreducible component of $X$ contains $g^{-1}\left(s_{0}\right)$.

Then $Y$ is reduced and $f \circ i: X_{\mathrm{red}} \rightarrow Y$ is an isomorphism. Moreover the open set of reduced points of $X$ contains the generic point of $g^{-1}\left(s_{0}\right)$. Assumption (a) implies Assumption (b) if $R$ is a localization of a finitely generated A-algebra, or if $A$ is a G-ring (cf. [4, § 34], or [3, § 32]). 
Start of Proof of Theorem 3. Let $\mathfrak{p}_{1}, \ldots, \mathfrak{p}_{m}$ be the minimal prime ideals of $A$. One has $A_{\mathfrak{p}_{i}}=k\left(\mathfrak{p}_{i}\right)$ for every $i$ since $A$ is reduced. Tensoring $0 \rightarrow A \rightarrow$ $\oplus_{i} A_{\mathfrak{p}_{i}}$ by $R$ one obtains by Assumption (b) that $R$ is reduced. Assumption (i) implies that $X \cong \operatorname{Spec} B$, where $B=\mathcal{O}_{X}(X)$ is a finite $R$-module and moreover $f^{\sharp}(Y): R \rightarrow B$ is injective, since $f$ is surjective and $R$ is reduced. The points of $X$ where $f$ is not flat form a closed subset $Z \subset X$. The image $f(Z)$ is closed in $Y$ since $f$ is finite. Let $Y^{\prime}=Y \backslash f(Z), X^{\prime}=f^{-1}\left(Y^{\prime}\right)$. Then $\left.f\right|_{X^{\prime}}: X^{\prime} \rightarrow Y^{\prime}$ is finite and flat.

We claim that $\zeta \in Y^{\prime}$. One applies $[4,20 . \mathrm{G}]$ to $\mathcal{O}_{S, s_{0}} \rightarrow \mathcal{O}_{Y, \zeta} \rightarrow \mathcal{O}_{X, \eta}$ and $M=\mathcal{O}_{X, \eta}$. By hypothesis $\mathcal{O}_{Y, \zeta}$ and $\mathcal{O}_{X, \eta}$ are flat $\mathcal{O}_{S, s_{0}}$-modules. Furthermore $Y_{s_{0}}$ is integral by Assumption (a), so $\mathcal{O}_{Y, \zeta} \otimes_{\mathcal{O}_{S, s_{0}}} k\left(s_{0}\right) \cong \mathcal{O}_{Y_{s_{0}}, \zeta} \cong k(\zeta)$ is a field. Hence $\mathcal{O}_{X, \eta} \otimes_{\mathcal{O}_{S, s_{0}}} k\left(s_{0}\right)$ is a flat $\mathcal{O}_{Y, \zeta} \otimes_{\mathcal{O}_{S, s_{0}}} k\left(s_{0}\right)$-module. Therefore $\mathcal{O}_{X, \eta}$ is a flat $\mathcal{O}_{Y, \zeta}$-module. The hypothesis that $g^{-1}\left(s_{0}\right)$ is irreducible implies that $f^{-1}(\zeta)=\eta$, therefore $\zeta \in Y^{\prime}, \eta \in X^{\prime}$.

Assumption (i) and Assumption (v) imply that every irreducible component of $Y$, being an image of some irreducible component of $X$, contains $h^{-1}\left(s_{0}\right)$ and in particular $\zeta$. Therefore the open set $Y^{\prime}$ is connected and dense in $Y$. We claim that $\left.f\right|_{X^{\prime}}: X^{\prime} \rightarrow Y^{\prime}$ is an isomorphism. First, $f_{*} \mathcal{O}_{X^{\prime}}$ is a locally free sheaf of a certain rank $d \geq 1$, since $\left.f\right|_{X^{\prime}}$ is finite, surjective and flat, and $Y^{\prime}$ is connected. One has $d=\operatorname{dim}_{k(y)} \Gamma\left(X_{y}, \mathcal{O}_{X_{y}}\right)$ for every $y \in Y^{\prime}$. Let $y=\zeta$. Then $f^{-1}(y)=\eta$ and furthermore $f: X \rightarrow Y$ is unramified at $\eta$. Indeed, it suffices to verify that $\left.f\right|_{X_{s_{0}}}: X_{s_{0}} \rightarrow Y_{s_{0}}$ is unramified at $\eta$. This holds since $\mathcal{O}_{Y_{s_{0}}, \zeta}=k(\zeta)$, $\mathcal{O}_{X_{s_{0}}, \eta}=k(\eta)$, for by hypothesis $X_{s_{0}}$ is irreducible and generically reduced, and furthermore $k(\zeta) \rightarrow k(\eta)$ is an isomorphism by Assumption (iv). We obtain that for $y=\zeta, X_{y}=\operatorname{Spec} k(\eta)$ and $d=\operatorname{dim}_{k(y)} \Gamma\left(X_{y}, \mathcal{O}_{X_{y}}\right)=1$. This shows that the morphism of sheaves of rings $f^{\sharp}: \mathcal{O}_{Y^{\prime}} \rightarrow f_{*} \mathcal{O}_{X^{\prime}}$ makes $f_{*} \mathcal{O}_{X^{\prime}}$ a locally free $\mathcal{O}_{Y^{\prime}}$-module of rank 1 . This implies that $f^{\sharp}: \mathcal{O}_{Y^{\prime}} \rightarrow f_{*} \mathcal{O}_{X^{\prime}}$ is an isomorphism, hence $\left.f\right|_{X^{\prime}}: X^{\prime} \rightarrow Y^{\prime}$ is an isomorphism. Since $Y$ is reduced this implies that the open set of reduced points of $X$ contains $X^{\prime}$, in particular the generic point $\eta$ of $g^{-1}\left(s_{0}\right)$ is a reduced point of $X$.

In order to prove the isomorphism $f \circ i: X_{\text {red }} \stackrel{\sim}{\longrightarrow} Y$ we replace $X$ by $X_{\text {red }}$ and observe that all the assumptions of the theorem hold for $f \circ i=f_{\text {red }}$ : $X_{\text {red }} \rightarrow Y$ and $g_{\text {red }}: X_{\text {red }} \rightarrow S$. We may thus assume that $X=\operatorname{Spec} B$ is reduced, so by Assumption $(\mathrm{v}) g^{-1}\left(s_{0}\right) \subset V(\mathfrak{p})$, where $\mathfrak{p}$ is any associated prime ideal of $B$.

Let $I$ be the radical ideal $I=I\left(Y \backslash Y^{\prime}\right) \subset R$. We will prove below that if $I \neq R$, then $\operatorname{depth}(I, R) \geq 2$. Assuming this statement one proves that $f: X \rightarrow Y$ is an isomorphism as follows. Consider the exact sequence of finite $R$-modules

$$
0 \rightarrow R \stackrel{f^{\sharp}(Y)}{\longrightarrow} B \rightarrow Q \rightarrow 0 .
$$

Let $R^{\prime}$ be the image of $R$. By way of contradiction let us assume $Q \neq 0$. Let $J=\sqrt{ } \operatorname{ann}(Q)$. One has $V(J)=\operatorname{Supp} Q$, so $Y \backslash V(J) \subset Y^{\prime}$. The isomorphism $f^{-1}\left(Y^{\prime}\right) \stackrel{\sim}{\longrightarrow} Y^{\prime}$, proved above, shows that $Y^{\prime} \subset Y \backslash V(J)$. Therefore $I=$ $J \neq R$. The stated inequality $\operatorname{depth}(I, R) \geq 2$ implies by [3, Theorem 16.6] that $\operatorname{Ext}_{R}^{1}(Q, R)=0$. Hence (3) splits, $B \cong R^{\prime} \oplus Q^{\prime}$. Let $\mathfrak{p}=\operatorname{ann}_{R}(x)$ be 
an associated prime ideal of $Q$. Since $\operatorname{Ass}(Q) \subset \operatorname{Supp}(Q)=V(J)$ (cf. $\quad[3$, Theorem 6.5]), one has $\mathfrak{p} \supset I$. Let $\mathfrak{p}^{\prime} \subset R^{\prime}$ be the image of $\mathfrak{p}$ and let $x^{\prime} \in Q^{\prime}$ be the preimage of $x$. Since $\mathfrak{p}^{\prime} \cdot x^{\prime}=0$ the subset $\mathfrak{p}^{\prime} \subset B$ consists of zero divisors, so $\mathfrak{p}^{\prime} \subset P_{1} \cup \cdots \cup P_{m}$, where $P_{i}, i=1, \ldots, m$, are the associated prime ideals of $B$. Let $\mathfrak{p}_{i}=R \cap P_{i}$. Then $\mathfrak{p} \subset \mathfrak{p}_{1} \cup \cdots \cup \mathfrak{p}_{m}$, so $\mathfrak{p} \subset \mathfrak{p}_{j}$ for some $j$. Let $P=\sqrt{ }(\mathfrak{m} B)$. Assumption (v) means that $P_{i} \subset P$ for every $i$. Hence $R \cap P \supset \mathfrak{p}_{j} \supset \mathfrak{p} \supset I$. Since $P \in \operatorname{Spec} B$ and $R \cap P \in \operatorname{Spec} R$ are the same as $\eta \in X$ and $\zeta \in Y$ respectively, one obtains that $\zeta$ belongs to $V(I)=Y \backslash Y^{\prime}$. This contradiction shows that $Q=0$ and therefore $f^{\sharp}(Y): R \rightarrow B$ is an isomorphism. The isomorphism $f \circ i: X_{\text {red }} \rightarrow Y$ is proved.

We prove now the last statement of the theorem. Let $C$ be a finitely generated $A$-algebra. Let $T=\operatorname{Spec} C, u: T \rightarrow S=\operatorname{Spec} A$ be the morphism corresponding to $A \rightarrow C$. Suppose there is an $A$-isomorphism of $R$ with $\mathcal{O}_{T, z}$ for some $z \in T$. Let $j: Y \rightarrow T$ be the composition $Y \stackrel{\sim}{\longrightarrow} \operatorname{Spec} \mathcal{O}_{T, z} \longrightarrow T$. One has $u(z)=s_{0}$ and $\mathcal{O}_{T, z} \otimes_{\mathcal{O}_{S, s_{0}}} k\left(s_{0}\right) \cong \mathcal{O}_{T_{s_{0}}, z}$. Assumption (a) implies, according to [5, Corollaire II.5.10], that the fiber $T_{s_{0}}$ is smooth at $z$, so $u$ is smooth at $z$ by [6, Theorem VII.1.8]. Smoothness at a point is an open condition so we may, replacing $T$ by an affine neighborhood of $z$, assume that $u: T \rightarrow S$ is smooth. It is moreover surjective since the flat morphism $u$ is an open map. Every fiber $T_{s}, s \in S$ is geometrically regular. Let $s \in S, y \in h^{-1}(s), t=j(y)$. One has $\mathcal{O}_{S, s}$-isomorphisms

$$
\mathcal{O}_{Y_{s}, y} \cong \mathcal{O}_{Y, y} \otimes_{\mathcal{O}_{S, s}} k(s) \cong \mathcal{O}_{T_{s}, t}
$$

Therefore $\mathcal{O}_{Y_{s}, y}$ is geometrically regular. This means that $R \otimes_{A} k(\mathfrak{p})$ is geometrically regular ring for every $\mathfrak{p} \in \operatorname{Spec} A$, in particular Assumption (b) holds.

Suppose now that $A$ is a $G$-ring. Then $A$ is quasi-excellent (cf. [4, § 34]). Let $k=k(\mathfrak{m}), \mathfrak{n}_{0}=\mathfrak{n} / \mathfrak{m} R \subset R \otimes_{A} k$. Assumption (a) implies that $R \otimes_{A} k$ is formally smooth with respect to the $\mathfrak{n}_{0}$-adic topology (cf. [4, $\left.\S 28 . \mathrm{M}\right]$ Proposition). Hence by Théorèm 19.7 .1 of $[7, \mathrm{Ch} .0]$ the homomorphism $A \rightarrow R$ is formally smooth with respect to the $\mathfrak{m}$-adic and $\mathfrak{n}$-adic topologies of $A$ and $R$. A theorem of André [8] yields that $A \rightarrow R$ is a regular homomorphism, so $R \otimes_{A} k(\mathfrak{p})$ is geometrically regular for every $\mathfrak{p} \in \operatorname{Spec} A$. This implies Assumption (b).

Our next goal is to prove that $\operatorname{depth}(I, R) \geq 2$ provided $I \neq R$, a statement used in the proof of Theorem 3. It is proved in [1, Ch.0] Proposition 10.3.1 that, given a Noetherian local ring $(A, \mathfrak{m})$ and a homomorphism of fields $k(\mathfrak{m}) \rightarrow K$, there exists a Noetherian local ring $(B, J)$ and a flat local homomorphism $(A, \mathfrak{m}) \rightarrow$ $(B, J)$ such that $\mathfrak{m} B=J$ and $k(J)$ is isomorphic to $K$ over $k(\mathfrak{m})$. We include the proof of the following known fact since we could not find a reference.

Lemma 4. Let $(A, \mathfrak{m})$ and $(R, \mathfrak{n})$ be Noetherian local rings, and let $A \rightarrow R$ be a local flat homomorphism. Let $k=k(\mathfrak{m}), K=k(\mathfrak{n})$. Suppose $K$ is separable over $k$. Suppose $R \otimes_{A} k$ is a regular ring of dimension $n \geq 1$. Let $(B, J)$ and $A \rightarrow B$ be as above: the homomorphism is local and flat, $\mathfrak{m} B=J$ and $k(J)$ is $k$-isomorphic to $K$. Then there is an $A$-isomorphism $\hat{R} \cong \hat{B}\left[\left[T_{1}, \ldots, T_{n}\right]\right]$, where $\hat{R}$ is the $\mathfrak{n}$-adic completion of $R$ and $\hat{B}$ is the J-adic completion of $B$. 
Proof. Let us first consider the case where $k(\mathfrak{m}) \rightarrow k(\mathfrak{n})$ is an isomorphism and $(B, J)=(A, \mathfrak{m})$. We have a commutative diagram of faithfully flat homomorphisms (cf. [3, Theorem 22.4])

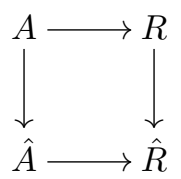

Let $t_{1}, \ldots, t_{n} \in \mathfrak{n}$ be elements such that $x_{i}=t_{i}(\bmod \mathfrak{m} R), i=1, \ldots, n$ generate the maximal ideal of the regular local $\operatorname{ring} R / \mathfrak{m} R \cong R \otimes_{A} k$. Let $\varphi$ : $\hat{A}\left[\left[T_{1}, \ldots, T_{n}\right]\right] \longrightarrow \hat{R}$ be the homomorphism of $\hat{A}$-algebras such that $\varphi\left(T_{i}\right)=t_{i}$ (cf. [9, Theorem 7.16]). Let $\hat{\mathfrak{m}}=\mathfrak{m} \hat{A}$ and $\hat{\mathfrak{n}}=\mathfrak{n} \hat{R}$ be the maximal ideals of $\hat{A}$ and $\hat{R}$. The ring $A^{\prime}=\hat{A}\left[\left[T_{1}, \ldots, T_{n}\right]\right]$ is local and complete with maximal ideal $M=\left(\hat{\mathfrak{m}}, T_{1}, \ldots, T_{n}\right)$ (cf. [10, Ch. III, $\S 2.6$, Proposition 6]). One has $\varphi(M)=\hat{\mathfrak{n}}$, so $\hat{R} / M \hat{R} \cong \hat{R} / \hat{\mathfrak{n}} \cong R / \mathfrak{n} \cong k(\mathfrak{n}) \cong k$. Hence $\varphi: A^{\prime} \rightarrow \hat{R}$ is surjective (cf. [3, Theorem 8.4]). In order to prove that $\varphi$ is injective, applying [3, Theorem 22.5], we need to verify that $\bar{\varphi}: A^{\prime} \otimes_{\hat{A}} k \rightarrow \hat{R} \otimes_{\hat{A}} k$ is injective. One has $\hat{R} \otimes_{\hat{A}} k \cong \hat{R} / \hat{\mathfrak{m}} \hat{R} \cong \widehat{R / \mathfrak{m} R}$. By assumption the composition $k=k(\mathfrak{m}) \rightarrow R / \mathfrak{m} R \rightarrow R / \mathfrak{n}=k(\mathfrak{n})$ is an isomorphism. Hence $\widehat{R / \mathfrak{m} R} \cong k\left[\left[x_{1}, \ldots, x_{n}\right]\right]$ (cf. [11, p. 124, Remark 2]). Therefore the composition

$$
k\left[\left[T_{1}, \ldots, T_{n}\right]\right] \stackrel{\sim}{\longrightarrow} A^{\prime} / \hat{\mathfrak{m}} A^{\prime} \stackrel{\bar{\varphi}}{\longrightarrow} \hat{R} / \hat{\mathfrak{m}} \hat{R} \stackrel{\sim}{\longrightarrow} k\left[\left[x_{1}, \ldots, x_{n}\right]\right]
$$

which transforms $T_{i}$ in $x_{i}$ is an isomorphism. This implies that $\bar{\varphi}: A^{\prime} \otimes_{\hat{A}} k \rightarrow$ $\hat{R} \otimes_{\hat{A}} k$ is an isomorphism. We conclude that $\varphi: \hat{A}\left[\left[T_{1}, \ldots, T_{n}\right]\right] \rightarrow \hat{R}$ is an isomorphism provided $k(\mathfrak{m}) \rightarrow k(\mathfrak{n})$ is an isomorphism.

Let us consider now the general case when $k=k(\mathfrak{m}) \rightarrow k(\mathfrak{n})=K$ is an arbitrary separable extension. By [3, Theorem 26.9] $K$ is 0 -smooth over $k$. Hence by [3, Theorem 28.10] $B$ is $J$-smooth over $A$. This implies that the homomorphism $B \rightarrow K=\hat{R} / \hat{\mathfrak{n}}$ has a lifting $\varphi: B \rightarrow \hat{R}$ which is a local homomorphism of $A$-algebras (see [3, p. 214]). Applying [4, 20.G] to $A \rightarrow B \rightarrow$ $\hat{R}$, taking into account that $B \otimes_{A} k \rightarrow \hat{R} \otimes_{A} k$ is flat since $B \otimes_{A} k \cong K$ is a field, we conclude that $B \rightarrow \hat{R}$ is flat. Furthermore $k(J)=B / J \rightarrow \hat{R} / \hat{\mathfrak{n}} \cong R / \mathfrak{n}=k(\mathfrak{n})$ is an isomorphism and by [3, Theorem 8.11$]$

$$
\hat{R} \otimes_{B} k(J) \cong \hat{R} \otimes_{B}\left(B \otimes_{A} k\right) \cong \hat{R} \otimes_{A} k \cong \hat{R} / \mathfrak{m} \hat{R} \cong \widehat{R / \mathfrak{m} R}
$$

is a regular local ring of dimension $n$. By the first part of the proof one concludes that $\hat{R} \cong \hat{B}\left[\left[T_{1}, \ldots, T_{n}\right]\right]$

Proposition 5. Let $(A, \mathfrak{m})$ and $(R, \mathfrak{n})$ be Noetherian local rings, and let $A \rightarrow R$ be a local flat homomorphism. Suppose $k=k(\mathfrak{m}) \rightarrow k(\mathfrak{n})=K$ is a separable extension. Suppose $R \otimes_{A} k$ is a regular ring. Let $I \subset R$ be a proper ideal such that:

a. none of the prime ideals of the set $A \cap V(I) \subset \operatorname{Spec} A$ is contained in an associated prime ideal of $A$; 


\section{b. $I \not \subset \mathfrak{m} R$.}

Then $\operatorname{depth}(I, R) \geq 2$.

Proof. We may replace $I$ by its radical and thus assume that $I=\sqrt{ } I$. Indeed, $\operatorname{depth}(I, R)=\operatorname{depth}(\sqrt{ } I, R)$ (see $[9$, Corollary 17.8]), $V(I)=V(\sqrt{ } I)$ and $I \not \subset$ $\mathfrak{m} R$ if and only if $\sqrt{ } I \not \subset \mathfrak{m} R$ since the condition that $R \otimes_{A} k$ is regular implies that $\mathfrak{m} R$ is a prime ideal. Furthermore Condition (b) implies that $\mathfrak{m} R \varsubsetneqq \mathfrak{n}$, so $\operatorname{dim} R \otimes_{A} k \geq 1$. Let $I=P_{1} \cap \cdots \cap P_{r}$, where $P_{i}, i=1, \ldots, r$ are the minimal prime ideals which contain $I$. We claim that there exists $a_{1} \in A \cap I$, such that $a_{1}$ is not a zero divisor of $A$. If this were not the case, then $A \cap I \subset \mathfrak{p}_{1} \cup \cdots \cup \mathfrak{p}_{s}$, where $\mathfrak{p}_{i}, i=1, \ldots, s$ are the associated primes of $A$. Then $A \cap I \subset \mathfrak{p}_{j}$ for some $j$ and consequently $A \cap P_{i} \subset \mathfrak{p}_{j}$ for some $i$ (cf. [11, Proposition 1.11]). This contradicts Condition (a).

Let $\hat{I}, \hat{R}$ be the $\mathfrak{n}$-adic completions of $I$ and $R$. The equality $\operatorname{depth}(I, R)=$ $\operatorname{depth}(\hat{I}, \hat{R})$ holds. Indeed, let $I=\left(x_{1}, \ldots, x_{m}\right)$. Consider the Koszul complex $K^{\bullet}=K^{\bullet}\left(x_{1}, \ldots, x_{m}\right)$, where $K^{i}\left(x_{1}, \ldots, x_{m}\right)=\Lambda^{i} N, N=\oplus_{i=1}^{m} R e_{i}$ and $d^{i}$ : $K^{i} \rightarrow K^{i+1}$ is $d^{i}(v)=x \wedge v$, where $x=\sum_{i=1}^{m} x_{i} e_{i}$. Then $\operatorname{depth}(I, R)=r$ iff $H^{i}\left(K^{\bullet}\right)=0$ for $i<r$ and $H^{r}\left(K^{\bullet}\right) \neq 0$ (cf. [9, Theorem 17.4]). Since $\hat{I}=I \hat{R}$ the images $x_{i}^{\prime}$ of $x_{i}$ in $\hat{R}, i=1, \ldots, m$, generate $\hat{I}$. The corresponding Koszul complex is $K^{\prime}=K^{\prime}\left(x_{1}^{\prime}, \ldots, x_{m}^{\prime}\right) \cong K^{\bullet}\left(x_{1}, \ldots, x_{m}\right) \otimes_{R} \hat{R}$. Since $R \rightarrow \hat{R}$ is faithfully flat one has that $H^{i}\left(K^{\prime}\right) \cong H^{i}\left(K^{\bullet}\right) \otimes_{R} \hat{R}$ and $H^{i}\left(K^{\prime} \bullet\right) \neq 0$ if and only if $H^{i}\left(K^{\bullet}\right) \neq 0$. By the above $\operatorname{criterion} \operatorname{depth}(I, R)=\operatorname{depth}(\hat{I}, \hat{R})$.

Let $(A, \mathfrak{m}) \rightarrow(B, J)$ and $\hat{R} \cong \hat{B}\left[\left[T_{1}, \ldots, T_{n}\right]\right]$ be as in Lemma 4 . The hypothesis $I \not \subset \mathfrak{m} R$ implies $\hat{I}=I \hat{R} \not \subset(\mathfrak{m} R) \hat{R}=\mathfrak{m} \hat{R}$ since $R \rightarrow \hat{R}$, being a faithfully flat homomorphism, has the property that $\mathfrak{a} \hat{R} \cap R=\mathfrak{a}$ for every ideal $\mathfrak{a}$ in $R$. Furthermore the ring extensions $A \rightarrow B \rightarrow \hat{B} \rightarrow \hat{R}$ yield $\mathfrak{m} \hat{R}=$ $(\mathfrak{m} B) \hat{R}=J \hat{R}=\hat{J} \hat{R}$. Therefore $\hat{I} \not \subset \hat{J} \hat{R}$.

Let $a_{1} \in A \cap I$ be a non zero divisor of $A$ as above. Let $a_{1}^{\prime} \in \hat{I}$ be its image in $\hat{R}$. Let $f \in \hat{I} \backslash \hat{J} \hat{R}$. We claim that $a_{1}^{\prime}, f$ is an $\hat{R}$-regular sequence. This implies that $\operatorname{depth}(\hat{I}, \hat{R}) \geq 2$. Abusing notation we identify $\hat{R}$ with $\hat{B}\left[\left[T_{1}, \ldots, T_{n}\right]\right]$. Let $\bar{f}=f(\bmod \hat{J} \hat{R}) \in K\left[\left[T_{1}, \ldots, T_{n}\right]\right]$. There exist positive integers $u_{1}, u_{2}, \ldots, u_{n}$ such that the automorphism $s$ of $K\left[\left[T_{1}, \ldots, T_{n}\right]\right]$ defined by $s\left(T_{i}\right)=T_{i}+T_{n}^{u_{i}}$ for $1 \leq i \leq n-1$ and $s\left(T_{n}\right)=T_{n}$ transforms $\bar{f}$ in $\bar{g}\left(T_{1}, \ldots, T_{n}\right)$ with $\bar{g}\left(0, \ldots, 0, T_{n}\right) \neq 0$ (cf. [10, Ch.VII $\S 3$ no.7] Lemma 3$)$. The same substitution yields a $\hat{B}$-automorphism $\varphi$ of $\hat{B}\left[\left[T_{1}, \ldots, T_{n}\right]\right]$. Let $g=\varphi(f)$. Let $C=\hat{B}\left[\left[T_{1}, \ldots, T_{n-1}\right]\right]$. This is a complete local ring with maximal ideal $\mathfrak{M}=$ $\left(\hat{J}, T_{1}, \ldots, T_{n-1}\right)$ and $\hat{R} \cong C\left[\left[T_{n}\right]\right]$. We claim that $a_{1}^{\prime}, g$ is a regular $\hat{R}$-sequence. Indeed, the injectivity of $A \stackrel{\cdot a_{1}}{\longrightarrow} A$ implies the injectivity of $\hat{R} \stackrel{\cdot a_{1}}{\longrightarrow} \hat{R}$ since the composition $A \rightarrow R \rightarrow \hat{R}$ is flat. One has $g(\bmod \mathfrak{M})=\bar{g}\left(0, \ldots, 0, T_{n}\right) \neq 0$ and $\hat{R} / a_{1}^{\prime} \hat{R} \cong\left(\hat{B} / a_{1}^{\prime} \hat{B}\right)\left[\left[T_{1}, \ldots, T_{n}\right]\right] \cong \bar{C}\left[\left[T_{n}\right]\right]$, where $\bar{C}=\left(\hat{B} / a_{1}^{\prime} \hat{B}\right)\left[\left[T_{1}, \ldots, T_{n-1}\right]\right]$ is a complete local ring with maximal ideal $\left(\hat{J} / a_{1}^{\prime} \hat{J}, T_{1}, \ldots, T_{n-1}\right)$. Applying [10, Ch.VII $\S 3$ no.8] Proposition 5 to the image of $g\left(\bmod a_{1} \hat{R}\right)$ in $\bar{C}\left[\left[T_{n}\right]\right]$ we conclude that $g\left(\bmod a_{1}^{\prime} \hat{R}\right)$ is not a zero divisor in $\hat{R} / a_{1}^{\prime} \hat{R}$. Therefore $a_{1}^{\prime}, g$ is a regular $\hat{R}$-sequence. Since $\varphi\left(a_{1}^{\prime}\right)=a_{1}^{\prime}, \varphi(f)=g$, the same holds for $a_{1}^{\prime}, f$ with $a_{1}^{\prime}, f \in \hat{I}$. We thus obtain that $\operatorname{depth}(I, R)=\operatorname{depth}(\hat{I}, \hat{R}) \geq 2$. 
END OF PROOF OF TheOREM 3. Recall that we have reduced the proof of the theorem to the case of reduced $X=\operatorname{Spec} B$ and we have assumed by way of contradiction that $I=I\left(Y \backslash Y^{\prime}\right) \varsubsetneqq R$. We prove that $\operatorname{depth}(I, R) \geq 2$ applying Proposition 5. The condition $I \not \subset \mathfrak{m} R$ is fulfilled since $\mathfrak{m} R=\zeta \in Y^{\prime}$. We want to verify that Condition (a) of Proposition 5 holds. By hypothesis $A$ is reduced, so one needs to prove that $A \cap V(I)$ contains none of the minimal prime ideals of $A$. Let $X_{i}=V\left(P_{i}\right), i=1, \ldots, n$ be the irreducible components of $X$. Assumption (i) and Assumption (v) imply, using that $\eta \in X^{\prime}$ and $\zeta=f(\eta) \in Y^{\prime}$, that there is a bijective correspondence between the irreducible components of $X$ and those of $Y$ given by

$$
X_{i} \mapsto X_{i} \cap X^{\prime} \mapsto f\left(X_{i} \cap X^{\prime}\right) \mapsto \overline{f\left(X_{i} \cap X^{\prime}\right)}=f\left(X_{i}\right)=Y_{i} .
$$

Let us give to $X_{i} \subset X$ and $Y_{i} \subset Y$ the structure of reduced closed subschemes and let $f_{i}: X_{i} \rightarrow Y_{i}$ be the morphism induced by $f, i=1, \ldots, n$. For every $i$ the affine schemes $X_{i}, Y_{i}$ are integral, the morphism $f_{i}$ is finite and if $x_{i} \in X_{i}$, $y_{i} \in Y_{i}$ are the generic points, the homomorphism

$$
\left(f_{i}^{\sharp}\right)_{y_{i}}: k\left(y_{i}\right)=\mathcal{O}_{Y_{i}, y_{i}} \longrightarrow \mathcal{O}_{X_{i}, x_{i}}=k\left(x_{i}\right)
$$

is an isomorphism since it coincides with $\mathcal{O}_{Y, y_{i}} \rightarrow \mathcal{O}_{X, x_{i}}$ and $y_{i} \in Y^{\prime}, x_{i}=$ $f^{-1}\left(y_{i}\right) \in X^{\prime}$. Let $Y^{\text {reg }}=\left\{u \in Y \mid \mathcal{O}_{Y, y}\right.$ is a regular ring $\}$. We claim that $Y^{\text {reg }} \subset Y^{\prime}$. Let $y \in Y^{\text {reg }}$. One has that $y \in Y_{i} \backslash \cup_{j \neq i} Y_{j}$ for some $i$. The regular ring $\mathcal{O}_{Y, y}=\mathcal{O}_{Y_{i}, y}$ is integrally closed in its field of fractions $k\left(y_{i}\right)$. Let $R_{i}=\mathcal{O}_{Y_{i}}\left(Y_{i}\right), B_{i}=\mathcal{O}_{X_{i}}\left(X_{i}\right), y=\mathfrak{q} \in \operatorname{Spec} R_{i}, S=R_{i} \backslash \mathfrak{q}$. The finite injective homomorphism of integral domains $f_{i}^{\sharp}\left(Y_{i}\right): \mathcal{O}_{Y_{i}}\left(Y_{i}\right) \rightarrow \mathcal{O}_{X_{i}}\left(X_{i}\right)$ induces an isomorphism of the fields of fractions $k\left(y_{i}\right) \stackrel{\sim}{\longrightarrow} k\left(x_{i}\right)$. Since $S^{-1} R_{i}$ is integrally closed one obtains that $S^{-1} R_{i} \rightarrow S^{-1} B_{i}$ is an isomorphism, hence $f_{i}^{-1}(y)$ con-

sists of a unique point $x \in X_{i}$ and $\left(f_{i}^{\sharp}\right)_{y}: \mathcal{O}_{Y_{i}, y} \rightarrow \mathcal{O}_{X_{i}, x}$ is an isomorphism. One has $f^{-1}(y)=f_{i}^{-1}(y)=\{x\}$ since $y \in Y_{i} \backslash \cup_{j \neq i} Y_{j}$. Furthermore $\mathcal{O}_{X_{i}, x}=\mathcal{O}_{X, x}$ since $X$ is reduced and $x \in X_{i} \backslash \cup_{j \neq i} X_{j}$. Therefore $\left(f^{\sharp}\right)_{y}: \mathcal{O}_{Y, y} \rightarrow \mathcal{O}_{X, x}$ is an isomorphism, so $y \in Y^{\prime}$. The claim that $Y^{\text {reg }} \subset Y^{\prime}$ is proved. Suppose now that $\mathfrak{q} \in V(I)=Y \backslash Y^{\prime}$ and $A \cap \mathfrak{q}=\mathfrak{p}$ is a minimal prime ideal of $A$. Let $S=A \backslash \mathfrak{p}$. One has by Assumption (b) that $R \otimes_{A} k(\mathfrak{p})=R \otimes_{A} A_{\mathfrak{p}}=S^{-1} R$ is a regular ring. Hence $R_{\mathfrak{q}}$ is a regular ring, so $\mathfrak{q} \in Y^{\text {reg }}$ which contradicts the inclusion $Y^{\text {reg }} \subset Y^{\prime}$ proved above. We thus prove that Condition (a) of Proposition 5 holds, therefore $\operatorname{depth}(I, R) \geq 2$. Theorem 3 is proved.

\section{A criterion for extending morphisms}

In this section we give the proofs of Theorem 1 and Theorem 2. We refer to $[12$, I. $\S 4.4]$ or $[5, \mathrm{II}]$ for the properties of smooth morphisms we use. We use an argument from the course notes of M. Mustata of 2009 for the proof of the following proposition, which we need.

Proposition 6. Let $g: X \rightarrow S$ be a morphism of algebraic varieties over an algebraically closed field $k$. Suppose there is an integer $d \geq 1$ such that for every 
$s \in S$ the fiber $g^{-1}(s)$ is irreducible of dimension $d$. Then every irreducible component of $S$ is dominated by a unique irreducible component of $X$ and every irreducible component of $X$ is a union of fibers of $g$. In each of the following three cases there is a bijective correspondence between the irreducible components of $X$ and those of $S$ given by $X_{i} \rightarrow \overline{g\left(X_{i}\right)}$ as well as by $S_{i} \rightarrow g^{-1}\left(S_{i}\right)$ in Case (a).

a. $g$ is a closed morphism.

b. All irreducible components of $X$ have the same dimension.

c. Every irreducible component of $X$ contains a point in which $g$ is flat.

Proof. Step 1. Let us first suppose that $S$ is irreducible. Let $X=X_{1} \cup \cdots \cup X_{n}$ be an irredundant decomposition of $X$ into irreducible components. Let $y \in S$. By hypothesis $g^{-1}(s)$ is irreducible, so $g^{-1}(s) \subset X_{j}$ for some $j$. The hypothesis implies that $g$ is surjective, consequently $S=\overline{g\left(X_{i}\right)}$ for some $i$. Renumbering we may suppose that $i=1$. Let $U=X_{1} \backslash \cup_{i>2} X_{i}$. If $x \in U$ and $s=g(x)$ then $X_{1}$ is the unique irreducible component of $X$ which contains $g^{-1}(s)$. The image $g(U)$ contains an open subset $V \subset S, V \neq \emptyset[13$, Theorem IV.3.7] and one has $g^{-1}(s) \subset X_{1}$ for every $s \in V$. We claim that $X_{i} \subset g^{-1}(S \backslash V)$ for $i \geq 2$. Let $x \in X_{i} \backslash X_{1}$. Then $s=g(x) \notin V$. The inclusion $X_{i} \backslash X_{1} \subset g^{-1}(S \backslash V)$ implies $X_{i} \subset g^{-1}(S \backslash V)$. Therefore $X_{1}$ is the unique irreducible component of $X$ which dominates $S$. Let $g_{1}=\left.g\right|_{X_{1}}$. If $s \in V$ then $g_{1}^{-1}(s)=g^{-1}(s)$, so $\operatorname{dim} g_{1}^{-1}(s)=d$. This implies that $\operatorname{dim} X_{1}-\operatorname{dim} S=d$ and $\operatorname{dim} g_{1}^{-1}\left(g_{1}(x)\right) \geq d$ for every $x \in X_{1}$ (ibid.). Let $x \in X_{1}, s=g(s)=g_{1}(s)$. Then $g_{1}^{-1}(s)$ is closed in $X$ of dimension $\geq d$ and moreover it is contained in $g^{-1}(s)$ which is by hypothesis irreducible of dimension $d$. Therefore $g_{1}^{-1}(s)=g^{-1}(s)$. This shows that $X_{1}$ is a union of fibers of $g: X \rightarrow S$.

Step 2. Let now $S$ be arbitrary algebraic variety. Let $X=X_{1} \cup \cdots \cup X_{n}$ $S=S_{1} \cup \cdots \cup S_{m}$ be the irredundant decompositions into irreducible components. Let $i \in[1, m]$. Since $g: X \rightarrow S$ is surjective $S_{i}=\overline{g\left(X_{j}\right)}$ for some $j$. Applying Step 1 to $S^{\prime}=S_{i}$ and $X^{\prime}=g^{-1}\left(S_{i}\right)$ one concludes that $X_{j}$ is the unique irreducible component of $X$ which dominates $S_{i}$. Let $X_{i}$ be an arbitrary irreducible component of $X$. Let $Z=\overline{g\left(X_{i}\right)}$. Applying Step 1 to $S^{\prime}=Z$ and $X^{\prime}=g^{-1}(Z)$ one obtains that $X_{i}$ is a union of fibers of $g: X \rightarrow S$.

Step 3. Case (a). If $g: X \rightarrow S$ is closed, then $S_{i}=g\left(X_{i}\right)$ is closed and irreducible and $X_{i}=g^{-1}\left(S_{i}\right)$. Hence $S=S_{1} \cup \cdots \cup S_{n}$ is an irredundant union of irreducible closed subsets of $S$.

Case (b). If $\operatorname{dim} X_{i}=N$ for every $i=1, \ldots, n$ then $\operatorname{dim} \overline{g\left(X_{i}\right)}=N-d$ for every $i=1, \ldots, n$. This implies that every $\overline{g\left(X_{i}\right)}$ is an irreducible component of $X$. By Step 2 every irreducible component of $S$ is dominated by a unique irreducible component of $X$, so $S=S_{1} \cup \cdots \cup S_{n}$ with $S_{i}=\overline{g\left(X_{i}\right)}$ is an irredundant decomposition of $S$ into irreducible components.

Case (c). The set of points $U \subset X$ in which $g$ is flat is open [4, Theorem 53]. By hypothesis $U \cap X_{i} \neq \emptyset$ for every $i=1, \ldots, n$. Therefore $U_{i}=U \backslash \cup_{j \neq i} X_{j}$ is an open nonempty subset of $X$ contained in $X_{i}$. The flat morphisms are open maps [6, Theorem V.5.1], therefore $g\left(U_{i}\right)$ is an open subset of $S$ contained in the irreducible closed subset $\overline{g\left(X_{i}\right)}$. Therefore $S_{i}=\overline{g\left(X_{i}\right)}$ is an irreducible 
component of $S$. By Step $2 S=S_{1} \cup \cdots \cup S_{n}$ with $S_{i}=\overline{g\left(X_{i}\right)}$ is an irredundant decomposition of $S$ into irreducible components.

Proof of Theorem 1. One has to prove that for every $y \in Y$ there is an affine neighborhood of $y$ such that $f$ restricted to its affine preimage is an isomorphism. So, it suffices to prove Theorem 1 for affine varieties $X, Y$ and $S$. Let $E=A(X), D=A(Y), C=A(S)$. One may furthermore assume that Spec $D \rightarrow \operatorname{Spec} C$ is a smooth morphism of relative dimension $d$. In order to prove that the finite homomorphism $D \rightarrow E$ is an isomorphism it suffices to prove that for every maximal ideal $\mathfrak{m}_{y} \subset D$, if $T=D \backslash \mathfrak{m}_{y}$, the localization $T^{-1} D \rightarrow T^{-1} E$ is an isomorphism. Let $\mathfrak{m}_{y} \cap C=\mathfrak{m}_{s}, s=h(y)$ and let $U=$ $C \backslash \mathfrak{m}_{s}=T \cap C$. Taking into account that $E=A(X)$ is reduced, we prove that $T^{-1} D \rightarrow T^{-1} E$ is an isomorphism applying Theorem 3, where the local homomorphism $A \rightarrow R$ is $C_{\mathfrak{m}_{s}}=U^{-1} C \rightarrow T^{-1} D=D_{\mathfrak{m}_{y}}, B=T^{-1} E$ and the diagram (2) is

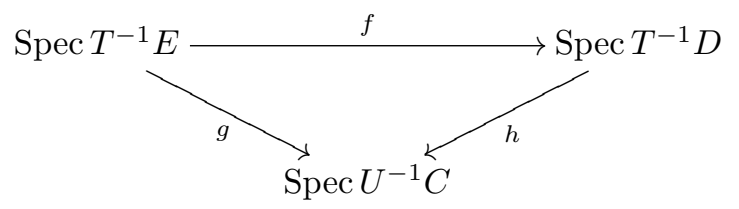

Let us verify the various assumptions of Theorem 3. Assumption (a) and Assumption (b) hold since Spec $D \rightarrow \operatorname{Spec} C$ is a smooth morphism and $k(\mathfrak{m})=$ $k=k(\mathfrak{n})$. Assumption (b) of Theorem 1 means that $D \rightarrow E$ is a finite homomorphism and $\operatorname{Spm} E \rightarrow \operatorname{Spm} D$ is surjective. This implies, since $D$ and $E$ are reduced, finitely generated algebras over $k$, that $D \rightarrow E$ is injective. By the goingup theorem $\operatorname{Spec} E \rightarrow \operatorname{Spec} D$ is surjective, hence $\operatorname{Spec} T^{-1} E \rightarrow \operatorname{Spec} T^{-1} D$ is surjective and this shows that Assumption (i) of Theorem 3 holds. By Assumption (c) of Theorem 1 the fiber $g^{-1}(s)$ is irreducible, hence there is a unique minimal prime ideal $P$ in $E$ which contains $\mathfrak{m}_{s} E, P=I\left(g^{-1}(s)\right)$. The existence of a point $x \in g^{-1}(s)$ such that $g$ is smooth at $x$ implies that $\operatorname{Spec} E \rightarrow \operatorname{Spec} C$ is smooth at $P$, hence the fiber $\operatorname{Spec} E / \mathfrak{m}_{s} E$ is smooth at the generic point $P / \mathfrak{m}_{s} E$, i.e. $E_{P} / \mathfrak{m}_{s} E_{P}$ is a field. This implies that the ideal $\mathfrak{m}_{s} E$ has an irredundant primary decomposition $\mathfrak{m}_{s} E=Q_{1} \cap Q_{2} \cap \ldots \cap Q_{m}$, where $Q_{1}=P$ is a prime ideal and $P \varsubsetneqq \sqrt{ } Q_{i}$ for $i \geq 2$. We claim that $P \cap D \subset \mathfrak{m}_{y}$. Indeed, by the surjectivity of $f: X \rightarrow Y$ there exists a point $x \in X$ such that $f(x)=y$, so $\mathfrak{m}_{x} \cap D \subset \mathfrak{m}_{y}$. One has $x \in g^{-1}(s)$, so $\mathfrak{m}_{x} \supset P$, therefore $P \cap D \subset \mathfrak{m}_{y}$. Localizing one obtains a primary decomposition

$$
\mathfrak{m}_{s} T^{-1} E=T^{-1} P \cap\left(\cap_{i \geq 2} T^{-1} Q_{i \geq 2}\right),
$$

where $T^{-1} P \varsubsetneqq \sqrt{ }\left(T^{-1} Q_{i}\right)$ for $i \geq 2$. This proves that $\operatorname{Spec} T^{-1} E / \mathfrak{m}_{s} T^{-1} E=$ Spec $B / \mathfrak{m} B$ is irreducible and generically reduced, so Assumption (ii) of Theorem 3 holds. Assumption (iii) for $\operatorname{Spec} T^{-1} E \rightarrow \operatorname{Spec} T^{-1} C$ follows from the smoothness of the morphism $\operatorname{Spec} E \rightarrow \operatorname{Spec} C$ at the point $P$ mentioned above. Assumption (iv) follows from Assumption (d) of Theorem 1. It remains 
to prove that Assumption (v) holds. The irreducible components of $\operatorname{Spec} T^{-1} E$ are of the form $V\left(T^{-1} P_{0}\right)$ where $P_{0}$ is a minimal prime ideal of $E$ such that $P_{0} \cap T=\emptyset$, i.e. $P_{0} \cap D \subset \mathfrak{m}_{y}$. By the going-up theorem there is a prime ideal $\mathfrak{p}^{\prime}$ in E such that $P_{0} \subset \mathfrak{p}^{\prime}$ and $\mathfrak{p}^{\prime} \cap D=\mathfrak{m}_{y}$. Hence $\mathfrak{p}^{\prime}=\mathfrak{m}_{x}$ for some $x \in X$ with $f(x)=y$. This implies that the irreducible component $V\left(P_{0}\right)$ of $X$ contains a point $x$ of the fiber $g^{-1}(s)$. The image of the smooth morphism $h: Y \rightarrow S$ is open. Let $S_{1}=h(Y)=g(X)$. The fibers of the morphism $g: X \rightarrow S_{1}$ are irreducible of dimension $d$ by hypothesis. Using Proposition 6 we conclude that the irreducible component $V\left(P_{0}\right)$ of $X$, being a union of fibers of $g: X \rightarrow S_{1}$, contains $g^{-1}(s)$, therefore $P_{0} \subset P$. Localizing one obtains $T^{-1} P_{0} \subset T^{-1} P$. Therefore $V\left(T^{-1} P_{0}\right)$ contains $V\left(T^{-1} P\right)$, which is the preimage of the closed point of the map $\operatorname{Spec} T^{-1} E \rightarrow \operatorname{Spec} U^{-1} C$. All the assumptions of Theorem 3 were verified, so we conclude that $T^{-1} D \rightarrow T^{-1} E$ is an isomorphism. Theorem 1 is proved.

Proof of Theorem 2. The morphism $f: X \rightarrow Y$ is proper and its image contains $U$, hence it is surjective. Furthermore the hypothesis of the theorem implies that $f$ has finite fibers, so $f$ is a finite morphism by Zariski's Main Theorem [14, Corollary 12.89]. One has the commutative diagram (1) with $g=h \circ f: X \rightarrow S$. We want to verify that the conditions of Theorem 1 hold. Condition (a) holds by hypothesis. Condition (b) was verified above. We claim that every fiber $g^{-1}(s), s \in g(X)=h(Y)$ is irreducible of dimension $d$. For the proof of this statement we may replace $S$ by its open subset $h(Y)$, so we may assume that $h: Y \rightarrow S$ is surjective. The fibers of $h$ are irreducible of dimension $d$, so according to Proposition 6(c) the irredundant decompositions of $S$ and $Y$ as finite union of closed irreducible subsets are respectively $S=\cup_{i=1}^{m} S_{i}$ and $Y=\cup_{i=1}^{m} Y_{i}$, where $S_{i}=\overline{f\left(Y_{i}\right)}, \operatorname{dim} Y_{i}-\operatorname{dim} S_{i}=d$, and furthermore every $Y_{i}$ is a union of fibers of $h: Y \rightarrow S$. Let $\Gamma_{i} \subset Y \times_{S} P$ be the graph of $\left.\varphi\right|_{Y_{i} \cap U}: Y_{i} \cap U \rightarrow P$. Then $\Gamma=\cup_{i=1}^{m} \Gamma_{i}$ and the irredundant decomposition of $X=\bar{\Gamma}$ is $X=\cup_{i=1}^{m} X_{i}$, where $X_{i}$ is the closure of $\Gamma_{i}$ in $Y \times_{S} P$. One has $X \cap\left(U \times{ }_{S} P\right)=\Gamma$, so $f^{-1}(U)=\Gamma$. Let $Z$ be an irreducible component of $g^{-1}(s)$. One has $Z \subset X_{i}$ for some $i$, hence $s=g(Z) \in S_{i}$ and $\operatorname{dim} Z \geq \operatorname{dim} X_{i}-\operatorname{dim} S_{i}=$ $\operatorname{dim} Y_{i}-\operatorname{dim} S_{i}=d$. Since $f: X \rightarrow Y$ is finite and $f(Z) \subset h^{-1}(s)$ one has that $f(Z)=h^{-1}(s)$. The map $\left.f\right|_{\Gamma}: \Gamma \rightarrow U$ is an isomorphism, so $Z$ contains the nonempty open subset $f^{-1}\left(U \cap h^{-1}(s)\right)$, hence $Z$ equals its closure. This shows that $g^{-1}(s)$ is irreducible for every $s \in g(X)=h(Y)$. The remaining conditions of (c) and (d) of Theorem 1 hold since $f^{-1}(U) \rightarrow U$ is an isomorphism. By Theorem $1 f: X \rightarrow Y$ is an isomorphism. The morphism $\tilde{\varphi}=\pi_{2} \circ f^{-1}: Y \rightarrow P$ is the extension of $\varphi: U \rightarrow P$. Theorem 2 is proved.

Acknowledgements. The author is grateful to J. Kollár for useful discussions. The author was on leave of absence from the Institute of Mathematics and Informatics of the Bulgarian Academy of Sciences. 


\section{References}

[1] A. Grothendieck, Éléments de géométrie algébrique. III. Étude cohomologique des faisceaux cohérents. I, Publications Mathématiques de l'IHÉS (11) (1961) 5-167.

[2] J. Kollár, Flatness criteria, J. Algebra 175 (2) (1995) 715-727. doi:10.1006/jabr.1995.1209.

[3] H. Matsumura, Commutative ring theory, Vol. 8 of Cambridge Studies in Advanced Mathematics, Cambridge University Press, Cambridge, 1986.

[4] H. Matsumura, Commutative algebra, Vol. 56 of Mathematics Lecture Note Series, Benjamin/Cummings, 1980, second edition.

[5] A. Grothendieck, Revêtements étales et groupe fondamental (SGA1), Vol. 224 of Lecture Notes in Mathematics, Springer-Verlag, 1971.

[6] A. Altman, S. Kleiman, Introduction to Grothendieck duality theory, Vol. 146 of Lecture Notes in Mathematics, Springer-Verlag, 1970.

[7] A. Grothendieck, Éléments de géométrie algébrique. IV. Étude locale des schémas et des morphismes de schémas. I, Publications Mathématiques de l'IHÉS (20) (1964) 5-259.

[8] M. André, Localisation de la lissité formelle, Manuscripta Math. 13 (1974) 297-307. doi:10.1007/BF01168230.

[9] D. Eisenbud, Commutative algebra With a view toward algebraic geometry, Vol. 150 of Graduate Texts in Mathematics, Springer-Verlag, 1995. doi:10.1007/978-1-4612-5350-1.

[10] N. Bourbaki, Commutative algebra. Chapters 1-7, Elements of Mathematics (Berlin), Springer-Verlag, 1998, translated from the French.

[11] M. F. Atiyah, I. G. Macdonald, Introduction to commutative algebra, Addison-Wesley, 1969.

[12] M. Demazure, P. Gabriel, Groupes algébriques. Tome I: Géométrie algébrique, généralités, groupes commutatifs, Masson \& Cie, Éditeur, Paris; North-Holland Publishing Co., Amsterdam, 1970.

[13] D. Perrin, Algebraic geometry, Universitext, Springer-Verlag London, Ltd., London; EDP Sciences, Les Ulis, 2008, an introduction, Translated from the 1995 French original by Catriona Maclean.

[14] U. Görtz, T. Wedhorn, Algebraic geometry I, Advanced Lectures in Mathematics, Vieweg + Teubner, 2010. doi:10.1007/978-3-8348-9722-0. 